\title{
Non-destructive Diagnosis of Kidney Cancer on 18- Gauge Core Needle Renal Biopsy using Dual-color Fluorescence Structured Illumination Microscopy
}

\author{
James Liu ${ }^{\mathrm{a}}$, Mei Wang ${ }^{\mathrm{b}}$, David Tulman ${ }^{\mathrm{b}}$, Sree H Mandava ${ }^{\mathrm{a}}$, Katherine N Elfer ${ }^{\mathrm{b}}$, Andrew \\ Gabrielson $^{\mathrm{a}}$, Weil Lai ${ }^{\mathrm{a}}$, Caleb Abshire ${ }^{\mathrm{a}}$, Andrew B Sholl ${ }^{\mathrm{c}}$, J Quincy Brown ${ }^{\mathrm{b}}$, and \\ Benjamin R Lee ${ }^{\mathrm{d}}$ \\ ${ }^{a}$ Department of Urology, Tulane University School of Medicine, New Orleans LA \\ ${ }^{b}$ Department of Biomedical Engineering, Tulane University, New Orleans LA \\ ${ }^{c}$ Department of Pathology and Laboratory Medicine, Tulane University School of \\ Medicine, New Orleans LA \\ ${ }^{\mathrm{d}}$ Division of Urology, University of Arizona College of Medicine, Tucson AZ \\ Word Counts:
}

Abstract: 235

Text: 2462

\section{Corresponding Author:}

Benjamin R. Lee, MD

Professor and Chief

Division of Urology

University of Arizona College of Medicine, Tucson, AZ 85724

Tel: 5206266014

Fax: 5206264933

Email: brlee@surgery.arizona.edu

Attributions: None

\section{Grant Numbers and Sources of Support:}

NIH NCI R21CA159936

NIH NCI R33CA196457

Institutional funding from the Tulane University School of Science and Engineering Katherine Elfer -National Science Foundation Graduate Research Fellowship Program David Tulman - National Science Foundation IGERT Fellowship (DGE-1144646)

Keywords: kidney biopsy, structured illumination microscopy (SIM), non-destructive, renal cell carcinoma 


\begin{abstract}
:
Objective:

To present a novel imaging technique used for rapid, non-destructive histological assessment of renal neoplasias using a dual-component fluorescence stain and structured illumination microscopy (SIM).

Methods:

After IRB approval, 65 total biopsies were obtained from 19 patients undergoing partial or radical nephrectomy. Biopsies were stained with a dual-component fluorescent, and optically sectioned SIM images were obtained from the surface of the intact biopsies. Specimens were subsequently fixed and analyzed using hematoxylin and eosin (H\&E) histopathologic methods and compared with SIM images. A single, board-certified pathologist blinded to specimens reviewed all SIM images and H\&E slides and determined the presence or absence of neoplasias. Results of blinded diagnosis of SIM were validated against traditional pathology.

Results:

Of the 19 patients, 15 underwent robotic partial nephrectomy and 4 underwent laparoscopic nephrectomies. Indications included clinical suspicion of renal cell carcinoma. In total, 65 biopsy specimens were available for review. 21 specimens were determined to be neoplastic on $\mathrm{H} \& \mathrm{E}$, while 41 represented benign renal tissue. The final sensitivity and specificity of our study was $79.2 \%$ and $95.1 \%$, respectively.

Conclusions:

SIM is a promising technology for rapid- near-patient ex-vivo renal biopsy assessment. By improving the ability to rapidly assess sufficiency of biopsy specimens and enabling immediate diagnostic capability, SIM aids in more effective biopsy performance, tissue triage, and patient counseling regarding management options. Additionally, because tissue is preserved, effective utilization of downstream diagnostic tests and molecular assessments are possible.
\end{abstract}




\section{Introduction:}

The role of percutaneous renal-core biopsy analysis of localized small renal masses $(<4 \mathrm{~cm})$ has continuously evolved in recent years. Historically, renal biopsy has been overshadowed by traditional imaging analysis like computed tomography and magnetic resonance imaging in surgical management guidance ${ }^{1}$. However, imaging modalities have demonstrated limited accuracy in characterization and prognosis of localized small renal masses when compared to histopathological analysis of sectioned specimens ${ }^{2}$. Cross-sectional imaging is effective in detecting the presence of a small renal mass but is unable to distinguish malignancy from a benign tumor (ie. oncocytoma) in a majority of these cases ${ }^{3}$. Additionally, with the rise of nephron-sparing surgery (NSS) and point-of-procedure histopathology assessment, there is a resurgence of interest in renal biopsy in the clinical management of patients with small renal tumors ${ }^{4}$. Significant interest has developed in comprehensive molecular evaluation of renal cell carcinoma, yet translation of these efforts to change patient management has yet to be fully defined. Overall, $78.7 \%$ of renal tumors present with a genomic alteration considered clinically relevant ${ }^{5}$.

Renal core biopsy analysis is the gold standard for histological analysis of these masses, however there are numerous shortcomings in its preparation. The process of fixation/freezing, sectioning and staining with Hematoxylin and Eosin (H\&E) destroys the integrity of the specimen and evaluation by a pathologist is typically performed at least 24 hours after specimen acquisition. Touch preparations can be useful, however without a dedicated cytopathologist to perform onsite assessment, this method will require additional time and cost, without concomitant improvement in efficacy. Unfortunately, this time consuming approach becomes logistically 
difficult to obtain point-of-procedure histopathology. Likewise, the limited tissue collected on core biopsy makes frozen section virtually impossible as a method for rapid diagnosis. Sufficiency of tissue from biopsy is also a reoccurring obstacle. Consequently, the current renal biopsy protocol is a lengthy, time-consuming process where patients sometimes are unable to obtain a definitive diagnosis and must return for additional biopsy procedures to further characterize the lesion.

Ex vivo imaging of fresh tissue specimens, stained with non-destructive fluorescent dyes is an attractive alternative to conventional biopsy modalities ${ }^{6}$. Previous studies done by our group have demonstrated the success of the ex vivo imaging modality, structured illumination microscopy (SIM), for rapid high-resolution diagnostic imaging of prostate biopsies ${ }^{7-8}$. SIM can be performed within minutes of tissue acquisition and produce images that closely recapitulate tissue histology visualized on traditional H\&E stained slides ${ }^{7}$. Likewise, by imaging fresh tissue we avoid the need for tissue processing, (e.g. fixation or freezing and sectioning) thereby preserving tissue for downstream definitive $\mathrm{H} \& \mathrm{E}$ analysis as well as any medically necessary additional diagnostic testing.

In this manuscript we demonstrate the potential of SIM for diagnosis of fresh, unfixed and unsectioned 18 gauge core needle biopsies for accurate, nondestructive diagnosis of fluorescently stained renal biopsies in point-of-procedure timeframes. We demonstrate that SIM can provide images with sufficient contrast and detail to allow delineation of benign vs. neoplastic renal tumors while streamlining clinical workflow. 


\section{Materials and Methods:}

Intact, core needle biopsies ( $\mathrm{n}=65$ ) from 19 patients receiving either robotic partial nephrectomy or laparoscopic radical nephrectomy were obtained under an Institutional Review Board protocol. Biopsies were taken ex vivo using a standard $18 \mathrm{G}$ core-needle biopsy technique (Bard Monopty, Bard, Tempe, AZ) from the renal neoplasm and adjacent benign renal parenchyma immediately after being removed from patient cavity. The biopsies were placed in saline and promptly mounted and scanned in the imaging suite within a half hour. The biopsies were then formalin fixed and prepared using standard H\&E.

The tissue staining and imaging protocol was adapted from prior work performed by Elfer et al ${ }^{9}$. Tissue specimens were prepared after being submerged in PBS post collection. Biopsies were submerged in Eosin Y solution 2\% v/v prepared in 80\% ethanol (E4009, Sigma-Aldrich, St. Louis, MO) for 10 seconds and then rinsed with PBS to remove excess stain. Excess fluid was removed from the biopsy with lab tissue and then submerged in $50 \mu \mathrm{M}$ DRAQ5 in PBS for 3 minutes (Biostatus, Shepshed, UK). In some cases, Eosin Y solution was reintroduced after the DRAQ5 step to increase the intensity of eosin staining. Biopsies were then patted with lab tissue to remove excess fluid and placed on slides for VR-SIM imaging (Figure 1).

The custom structured illumination microscope (SIM) has been described in detail previously ${ }^{7,8}$. In this work, the system was modified to enable imaging of the dual component stain, specifically by adding a $630 \mathrm{~nm}$ LED for DRAQ5 excitation (UHP-MIc-LED-630, Prizmatix, Southfield MI) in addition to the $475 \mathrm{~nm}$ LED for eosin excitation (Thorlabs, Newton, NJ). The two LEDS were combined using a dichroic beam combiner (Prizmatix, Southfield, MI). A 
ferroelectric spatial light modulator (SLM, 3DM, Forth Dimension Displays, Fife, UK) was used to project patterns for structured illumination microscopy onto the sample through a $10 \mathrm{X} 0.45$ NA Plan Apo objective lens (Nikon, Tokyo, Japan) in epi-illumination configuration. A multiband filter cube (Semrock, Rochester, NY) was used to allow excitation and emission of both DRAQ5 and eosin; the DRAQ5 and eosin images were taken sequentially at each frame position by illumination of respective LED. Samples were imaged through the 10X objective and Nikon tube lens onto a Hamamatsu Orca Flash 4.0 v2 scientific CMOS camera. Pattern illumination frequencies were chosen as described previously ${ }^{7}$, with an integration time of 100 ms-110 ms per frame. After imaging, biopsies were fixed in $10 \%$ formalin for a minimum of 48 hours before being sent to pathology for standard H\&E processing for comparison against SIM images. The eosin and DRAQ5 fluorescence SIM images were combined into a single pseudocolored image to approximate the appearance of $\mathrm{H} \& \mathrm{E}(\mathrm{D} \& \mathrm{E})$ as described by Bini et $\mathrm{al}^{9}$.

One board certified genitourinary pathologist co-author (ABS) reviewed all SIM images as a single cohort, and then reviewed the corresponding H\&E images as single cohort; both in a blinded fashion separated by 2 months to prevent recall bias. Slides were collected and reviewed in sets in order to optimize blinding protocol. SIM was reviewed as digital images that allow digital zoom. Pathologist diagnosed the biopsies as either neoplastic or benign and further characterized the neoplastic specimens into benign or malignant categories as needed. In addition, the pathologist rater also gave a confidence ranking of each diagnosis ranging from 1 to 5 where 1 indicated most likely to be benign, 2 indicated possibly benign, 3 indicated indeterminate between cancerous or benign, 4 indicated possibly malignant, and 5 indicated most likely to be malignant. H\&E slides were diagnosed using same grading method. 
SIM results were compared against H\&E prepared slides from the needle core biopsies. This comparison was done without incorporating pathology results of the final nephrectomy specimens to better simulate a true clinical scenario in which only the needle core biopsy would be available for histologic review.

\section{Results:}

An average of 3.6 samples/cores were taken from each of the 19 patients; 15 of which underwent robotic partial nephrectomy for clinical $\mathrm{T} 1 \mathrm{a} / \mathrm{b}$ renal masses, and 4 underwent laparoscopic radical/simple nephrectomy. Indications included clinical suspicion of renal cell carcinoma, as well as a patient with non-functioning kidney to determine assessment of medical renal disease.

Figure 1 contains a schematic and photograph of the SIM set up and a photograph of the biopsy prepared for imaging. Fresh 18 -gauge core biopsies were taken in the OR and core samples were brought to lab under sterile conditions for imaging.

Figure 2 shows a comparison of benign and malignant kidney using both traditional $\mathrm{H} \& \mathrm{E}$ stain and dual-color SIM imaging. Figure. 2 A presents a SIM image of a whole core biopsy using the pseudo H\&E (D\&E) preparation. Figure $2 \mathrm{~B}$ and D show H\&E sections while $\mathrm{C}$ and $\mathrm{E}$ show corresponding dual-stained (D\&E) SIM images. Benign renal parenchyma seen in Figure 2B and $\mathrm{C}$ show clear glomeruli with adjacent renal tubules and stroma. Clear cell carcinoma seen in 2D 
and E illustrates an abnormal proliferation of cells with clear cytoplasm and a definitive loss of normal renal parenchymal architecture.

Table 1 presents sensitivity, specificity, positive predictive value (PPV), negative predictive value (NPV), and accuracy (ACC) for the 65 biopsies imaged in this work (41 benign, 24 neoplastic). Test was determined by pathologist reading of D\&E compared to true disease determined by pathologist from H\&E interpretation. 39 out of 41 benign biopsies were correctly identified as benign, whereas 19 out of 24 biopsies were correctly identified as malignant. This resulted in a sensitivity of $79.1 \%$, a specificity of $95.1 \%$, for an overall diagnostic accuracy of $89.2 \%$ for rapid point-of-acquisition SIM imaging.

\section{Discussion:}

Over the past few decades, indications for renal biopsy in urology have included history of previous malignancy, bilateral renal masses, concern of lymphoma, as well as to rule out metastatic disease ${ }^{11}$. Traditionally, the above tumors initially present clinically with pertinent signs of malignant disease, whereas adjunct imaging serves a confirmatory role for the presence of disease. A biopsy with histologic subtyping of tumor in this setting would then direct subsequent management. However, with the advent of more robust imaging modalities with higher resolution, as well as their widespread application in the clinical setting, there has been an increase in incidentally detected small renal masses. Imaging for the diagnosis of renal malignancy in these small tumors has been inconsistent. Several studies have demonstrated that when it comes to small renal masses, particularly those less than $<20 \mathrm{~mm}$, the accuracy of preoperative CT and MRI is significantly decreased ${ }^{2}$. In this setting, benign renal masses such as 
oncocytoma and/or angiomyolipoma may not be appropriate for radical surgical intervention. The diagnosis of malignancy, however, may become extremely important in these small masses, and the use of emerging small molecules for treatment may be effective. Thus, there has been a revival and new focus on the renal biopsy as a means of diagnosing malignancy early in these patients while also leveraging new molecular diagnostics to determine proper personalized therapeutics.

Renal biopsies have improved in the past few years with advances in biopsy technology as well as biopsy technique, however several limitations remain ${ }^{12-13}$. Non-diagnostic biopsy with insufficient sample usually warrants the patient to return to the clinic and undergo another procedure. This not only increases patient risk of hemorrhage, but delays diagnosis and treatment ${ }^{14}$. Alternatively, sufficient tissue may be collected for fundamental diagnostic purposes, but may be insufficient for further molecular and/or immunohistochemical testing. The above setbacks are compounded by the fact that current biopsy preparation techniques require formalin fixation, paraffin embedding and H\&E staining, which in most institutions takes greater than 24 hours processing time. This same process also destroys the tissue during the sectioning process, and can markedly limit downstream immunohistochemical and/or molecular studies. Therefore, there is an urgent need to improve real time ex vivo renal biopsy technology, interpretation, and protocol.

In this study we explored the potential of Structured Illumination Microscopy (SIM) in the diagnosis of renal neoplasia using 18-gauge fresh renal core biopsies. Previous studies by our group demonstrated efficacy of the SIM platform in the diagnostic imaging of prostate biopsies 
with a sensitivity ranging from $75 \%-92 \%$ and a specificity ranging from $78-89 \%{ }^{7}$. In this study we adapted a novel use of DRAQ5 and eosin (D\&E) staining, which provides pseudohistochemical imaging comparable to the industry standard hematoxylin and eosin without the destructive tissue preparation. Histology as seen in Figure 2 demonstrates the ability of rapid $e x$ vivo SIM for rendering diagnostic quality images while retaining tissue architectural features needed for accurate diagnosis.

Table. 1 presents the diagnostic accuracy of the in this biopsy cohort. The sensitivity and specificity were 79.2 and $95.1 \%$, respectively. It is important to note the purpose of the renal biopsy is to more effectively diagnose malignancy so patients with benign tumors will not undergo excessive procedures. Therefore, the high specificity paired with a high negative predictive value of $90.7 \%$ illustrate the efficacy of our platform in discriminating benign from malignant kidney pathology. Likewise, another benefit of SIM is that it allows for rapid diagnosis, as samples can be read at the time of procedure with minimal additional time needed for tissue preparation. This allows the urologist and the pathologist to determine if the biopsy taken is sufficient and provides a clear diagnosis in a time efficient manner. By providing rapid feedback and preserving tissue, SIM improves point of procedure patient care, helps the urologist determine management and clinical care, and provides the pathologist with unaltered diagnostic tissue available for standard processing, immunohistochemistry, as well as molecular diagnostic studies as needed.

In this pilot study, there were 5 false-negative and 2 false-positive calls out of 65 total calls. Our group is taking several steps to improve the sensitivity in future studies. Specifically, we are 
continuing to optimize the staining and imaging parameters to increase image quality, including experimenting with higher magnifications, which would provide clear sub-nuclear resolution, further enhancing identification of cancer cell type. In addition, as we continue to accrue SIM images of benign and malignant renal pathologies creating a clinical image atlas for pathologist training, we expect these types of diagnostic errors to decrease.

The future of cancer treatment will be built on personalized and point-of-care medicine. As our understanding of cancer molecular genetics continues to grow, downstream immunohistochemical studies and markers will become crucial in treatment decisions. ${ }^{15}$ The SIM and D\&E protocol is a promising step towards that future. The technology provides rapid diagnosis that does not interfere with downstream tissue integrity. Likewise, the DRAQ5 stain binds stoichiometrically to DNA. Therefore, D\&E staining can theoretically be used to measure DNA content in a tumor sample. This information would be immensely useful for any type of analysis requiring nuclear DNA content and/or integrity, including oft utilized PCR and FISH molecular testing.

This study was done at a single institution with 65 total samples collected from 19 different patients. SIM shows a promising future for real-time non-destructive renal biopsy imaging. Given these promising results for rapid evaluation of malignant and benign $18 \mathrm{G}$ core biopsies from post-nephrectomy specimens, further studies validating this protocol in actual in vivo tissue renal biopsy are warranted. Improvements in the imaging technology of SIM can be further enhanced and studies on DRAQ5, especially in the measurement of DNA content distribution among different renal malignancy subtypes will be explored. Though these advances greatly 
benefit urological procedures, the ability to accurately distinguish glomeruli on biopsies at the bedside can also have significant impact on practices in other fields such as nephrology, transplantation, and medical oncology.

\section{Conclusion:}

As the role of renal biopsy especially in the diagnosis and management of small renal masses increases, new developments and technology must be implemented to improve on limitations of tissue collection and stain preparation. The SIM platform and D\&E staining protocol is a promising way to approach renal biopsy that not only improves diagnostic turnaround, but also preserves tissue for downstream studies, thereby promoting point of care medicine.

\section{References:}

1. Gautam G, and Kevinc ZC. The Current Role of Renal Biopsy in the Management of Localized Renal Tumors Indian Journal of Urology Indian J Urol 2009; 25(4): 494

2. Dechet CB, Zincke H, Sebo TJ, et al. Prospective analysis of computerized tomography and needle biopsy with permanent sectioning to determine the nature of solid renal masses in adults." J Urol 2003; 169(1): 71-4

3. Davidson AJ, Hayes WS, Hartman DS, et al. Renal Oncocytoma and Carcinoma: Failure of Differentiation with CT. Radiology 1993; 186(3): 693-96

4. Benway BM and Bhayani SB. Approach to the Small Renal Mass: Weighing Treatment Options. Current Urology Reports Curr Urol Rep 2009; 10(1): 11-16 
5. Murray B, Albiges L, Van Allen EM et al. Precision medicine approach in kidney cancer: a pan renal cell carcinoma (RCC) study across three cancer genome atlas (TCGA) datasets for clinically relevant target identification. J Clin Oncol 2015; 33: 11-13

6. Dobbs JL, Ding H, Benveniste AP, et al. Feasibility of Confocal Fluorescence Microscopy for Real-time Evaluation of Neoplasia in Fresh Human Breast Tissue. J. Biomed. Opt Journal of Biomedical Optics 2013; 18(10): 106-16

7. Wang M, Kimbrell HZ, Sholl AB, et al. High-Resolution Rapid Diagnostic Imaging of Whole Prostate Biopsies Using Video-Rate Fluorescence Structured Illumination Microscopy. Cancer Research 2015; 75(19): 4032-041.

8. Schlichenmeyer TC, Wang M, Elfer KN, and Brown JQ. Video-rate Structured Illumination Microscopy for High-throughput Imaging of Large Tissue Areas. Biomedical Optics Express Biomed. Opt. Express 2014; 5(2): 366

9. Elfer KN, Sholl AB, Kimbrell HZ, et al. A topical fluorescent analogue for virtual hematoxylin and eosin histology in point-of-care ex vivo microscopy. European Conferences on Biomedical Optics 2015; Munich, Germany: International Society for Optics and Photonics.

10. Bini J, Spain J, Nehal K, et al. Confocal mosaicing microscopy of human skin ex vivo: spectral analysis for digital staining to simulate histology-like appearance. J Biomed Opt 2011;16(7)

11. Anik SV. Review: Biopsy of Renal Masses: When and Why. Cancer Imaging 2009; 9(1): $44-55$

12. Andonian S, Okeke Z, Okeke DA, et al. Number of needle passes does not correlate with the diagnostic yield of renal FNAC. J Endourol 2008; 22(10): 2377-2380 
13. Andonian S, Okeke Z, VanderBrink BA, et al. Aetiology of non-diagnostic renal fineneedle aspiration cytologies in a contemporary series. BJU Int 2009 103(1): 28-32

14. Maturen KE, Nghiem HV, Caoili EM et al. Renal Mass Core Biopsy: Accuracy and Impact on Clinical Management. American Journal of Roentgenology 2007; 188(2): 56370

15. Gagan J and Van Allen EM. Next-generation Sequencing to Guide Cancer Therapy. Genome Medicine Genome Med 2015; 7(1)

\section{Figures Legends}

Figure 1. Schematic and photograph of SIM set-up

Figure 2. Comparison of H\&E and D\&E imaging of benign and malignant kidney tissue. Figure $2 \mathrm{~A}$ illustrates SIM of whole core biopsy. Figure 2B and $\mathrm{C}$ illustrate $H \& E$ and $D \& E$ of benign renal sections, respectively. Figure $2 D$ and $2 E$ illustrate $H \& E$ and D\&E of malignant renal sections, respectively. 

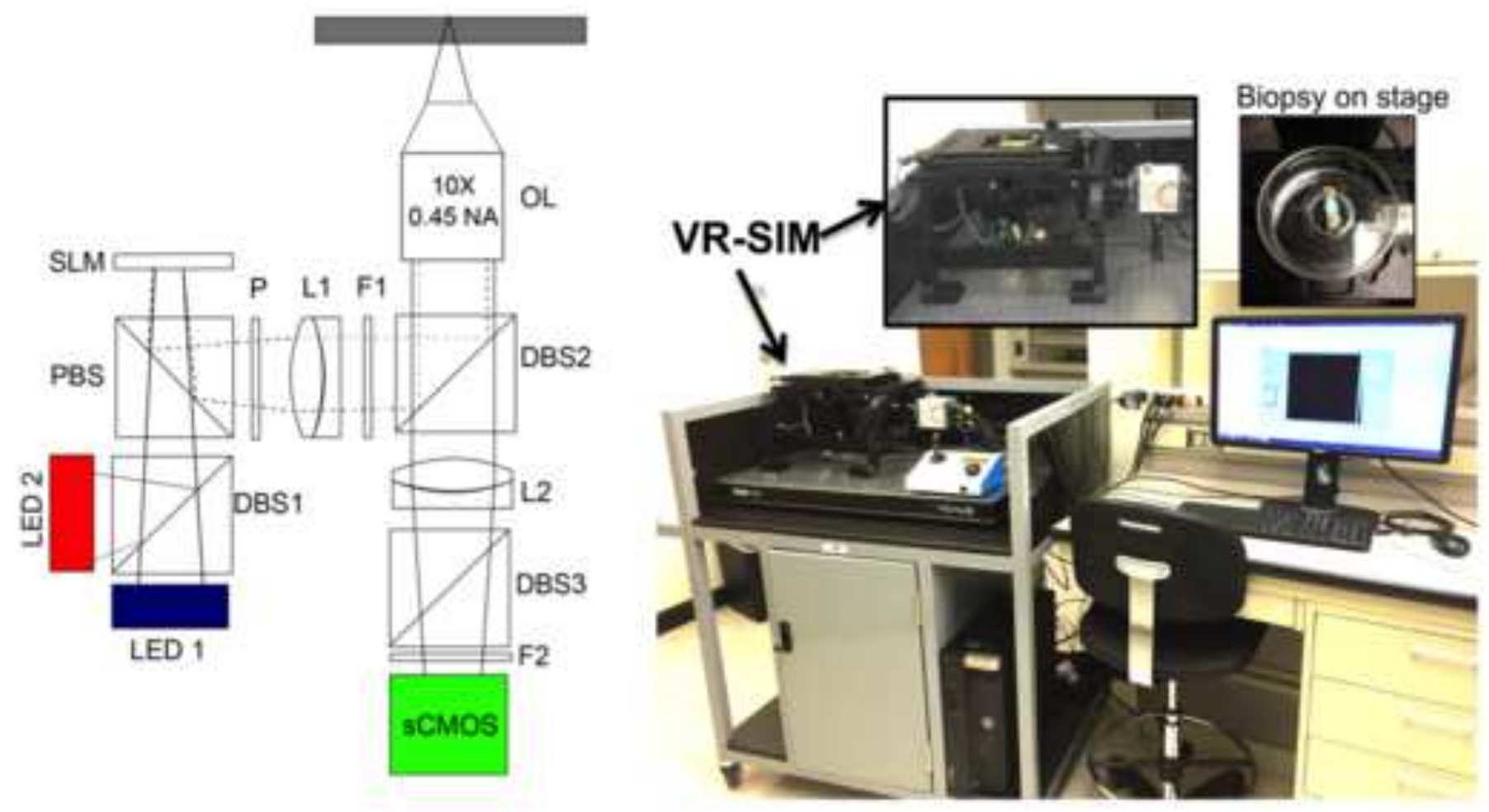

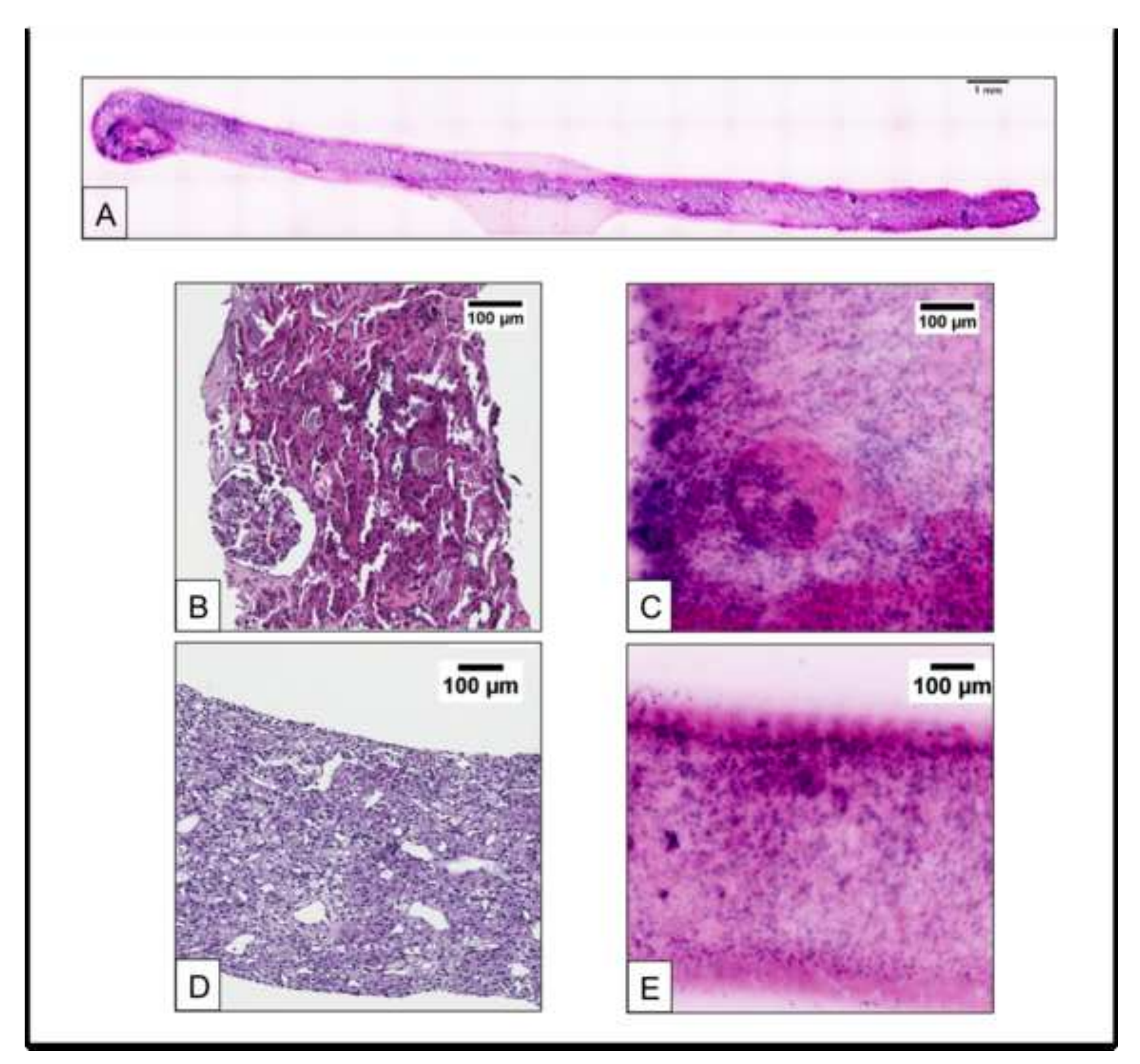

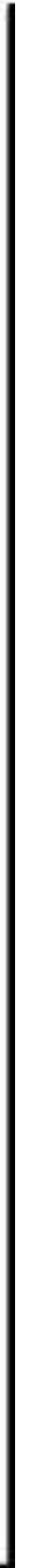

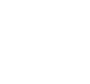




\begin{tabular}{|l|l|l|l|l|}
\hline Sensitivity & Specificity & PPV & NPV & ACC \\
\hline 0.792 & 0.951 & 0.826 & 0.907 & 0.892 \\
\hline
\end{tabular}

Table 1. Summary of sensitivity, specificity, positive predictive value, negative predictive value, and accuracy for 65 biopsies 